\title{
A Method for Weakening Residual Errors in Multi-beam Swath Joins based on Terrain Spectrum Analysis
}

\author{
WANG Shengping ${ }^{1,2,3^{*}}$, ZHOU Ping ${ }^{1,2}$, WU Ziyin ${ }^{3}$, WANG Jianqiang ${ }^{1,4}$ and CHEN Hanqing ${ }^{1,2}$ \\ ${ }^{1}$ Faculty of Geomatics, East China University of Technology, Nanchang 330013, China \\ ${ }^{2}$ Key Laboratory of Watershed Ecology and Geographical Environment Monitoring, East China University of Technology, Nanchang \\ 330013, China \\ ${ }^{3}$ Key Laboratory of Submarine Geosciences, Second Institute of Oceanography, Hangzhou 310012, China \\ ${ }^{4}$ School of Mathematical and Geospatial Sciences, RMIT University, Melbourne VIC 3000, Australia
}

Received 13 April 2016; Accepted 16 July 2016

\begin{abstract}
Some factors lead to spurious signals in the multi-beam sounding process, including complex changes of the sea, the vessel motion attitede, the unreasonable setting of equipment parameters and so on. Although these factors were filtered strictly and the system errors were compensated in the post processing, residual errors still exist in multi-beam swath joins. Such errors produce uplift and abnormal phenomena in the measured terrain between swaths. Therefore, a new method of terrain spectrum analysis was established to improve the efficiency of massive data processing and to accurately reflect the submarine topography. This method was based on the blend of extraction technology of short-wave signals and fitting model of long-wave signals, among which wavelet analysis was used to extract the terrain highfrequency signals (short-wave) and multi-surface function was applied to fit the long-wave signals. Then, the two types of signals were fused to reconstruct the actual seabed topography. Finally, the indexes of depth discrepancy were used to estimate the effect of residual errors. Experimental results show that the proposed method could effectively eliminate the uplift phenomenon of the seabed Digital Elevation Model (DEM), and correctly reflect the detailed topographical variation. This work could further improve the sounding accuracy and the efficiency of swath joins, and beneficial to the fusion of multi-beam digital signals and the image information of side scan sonar.
\end{abstract}

Keywords: Swath joins, Terrain spectrum analysis, Depth discrepancy, Residual error weaken

\section{Introduction}

Multi-beam swath sounding system is a highly integrated type of seafloor measuring equipment that offers full coverage, high precision, high density, and high efficiency [1-3]. This system is primarily used to detect the seabed topography of large areas. In process of full coverage measurement, swath joins are important link to ensure the accuracy of seabed terrain [4-6]. Thus, several new and efficient methods have been proposed to deal with these errors, such as sound velocity, vessel posture and installation deviation. However, induced residual errors continue to exist after the above error processing. Inevitably, swath joins bring a systematic influence on the water depth calculation and result in a "crying face" or "smile" phenomenon in the measured terrain. How to detect and weaken systematic residual errors will be an urgent need to solve in underwater topographic survey.

\section{State of the Art}

Data processing in swath joins is part of the adjustment of seabed sounding, and the quality of joins directly affects the mapping precision of edge beam. To obtain the high

\footnotetext{
*E-mail address: shpwang@ecit.edu.cn

ISSN: $1791-2377$ @ 2016 Eastern Macedonia and Thrace Institute of Technology. All rights reserved.
}

accuracy of marine surveyed results, thorough research on the processing of sounding data must be conducted [7-10]. However, a prominent problem is how to detect systematic errors and assess the accuracy of sounding. Moreover, residual errors in the swath joins continue to exist after system error detection. Therefore, the technology of swath joins in the seabed is first introduced in the following parts of this section. The theories and methods of system error detection in recent years are then described. Finally, some studies on residual error weakening are reviewed.

The error propagation method of multi beam was deduced in 1995 [11]. On this basis, the Combined Uncertainty and Bathymetry Estimator (CUBE) algorithm was proposed [12], which by using the gridding and filtering to realize the swath joins. This algorithm has a low memory, runs faster and real-time processing. The least square algorithm was applied to reverse systematic deviation [13], but only applied to test the system before running tests. Moreover, a weighted least square error method was extended in multi-beam swath joins [14]. Their method can obtain a better match between sounding data, and also simplify the process of stitching.

A two-step filtering method was applied to eliminate systematic deviation between adjacent swaths [15]. This method takes advantage of the combined multi-beam and single-beam sounding data. The two-step processing can be used to simplify the calculation, and the swaths can be processed separately. However, the error equation is no 
longer rank-deficient, and the error correlation among swaths and the necessary check information are ignored. The adjustment model of swath network was introduced to detect system errors of Ping [16]. However, this method requires large amount of Ping data and imported parameters, thereby leading to a large-scale normal equation. Meanwhile, based on the Ping structure, a quick algorithm based on the sequential adjustment principle was constructed [17]. This algorithm is used to calculate the parameters of swath net. However, the number of gain matrix, the overlapping rate, and the sparseness of intersection point affect the applicability of this algorithm.

Using these adjustment models [15-17] as system error detection algorithms still fails to weaken induced residual errors. The effect of residual errors is systematic and comprehensive, which is difficult to be separated or weakened by conventional detection methods. Therefore, according to different error sources, the corresponding corrective measures should be taken to improve the quality of the data collection. Finally, the human-computer interaction is realized in a visual way, but actual residual errors on edge beams are insignificantly weakened [18].

In study [19-20], a method of weakening the residual errors based on terrain geometric rectification and the method of discrete Fourier transform (DFT) was proposed. Their model is based on Ping continuous matching process, and has difficulty weakening the residual errors in nonoverlapping areas. Moreover, long-wave signals were fitted by the planar or polynomial curved surface fitting method, which inability to reflect the complex variation of seabed topography accurately.

Thus, a method of weakening the residual errors based on terrain spectrum analysis is presented in this study. First, the bathymetric signal after filtering and system error processing is decomposed into a short-wave signal with high-frequency feature and a long-wave signal. Then, wavelet analysis is used to extract the feature of highfrequency signals as a short-wave. Meanwhile, multi-surface function method is also adopted to fit the terrain long-wave signals. Finally, the high-frequency and long-wave signals are combined to synthetize a new seabed signal. These reconstructed signals indicate that the residual errors are effectively weakened and can accurately reflect the seabed topography.

The rest of this paper is organized as follows. Section 3 describes the research route and details of the proposed algorithm. Section 4 presents Experiments A and B for evaluating the validity of the weakening effect of residual errors and for verifying the feasibility of the proposed algorithm. Section 5 elaborates the conclusions.

\section{Methodology}

\subsection{Prophase Quality Control of Bathymetric Data}

Complexity of the seabed and the deficiencies of the multibeam sounding system produce some gross errors in the process of measurement [21-22]. First, the method of Ware, Knight, and Wells is used to diagnose and calibrate the gross errors of original bathymetric data [23]. The sound velocity, vessel posture, and compensation measurement parameters are random, and thus, they create a systematic feature, especially in marginal beam areas. Therefore, the two-step filtering method can be used to further improve the point quality and eliminate or weaken the influence of system errors [24]. By combining the above-mentioned data processing methods, the corrected bathymetric data are obtained, which are considered the data base of the terrain spectrum analysis method in this study.

\subsection{Fundamental of Terrain Spectral Analysis}

The influence of residual errors changes the depth of longperiod signals (long-wave), whereas induce no effect on short-period signals of terrain micro variation (short-wave) [19]. Meanwhile, the data quality of central beam is considered credible. In other words, the main trend of submarine topography can be fitted by the central beam sounding data. Thus, a method for analyzing the residual error of the spectrum analysis is proposed. The method includes the wavelet analysis of short-wave signals and the multi-surface function fitting method of long-wave signals. The specific research route is shown in Fig. 1.

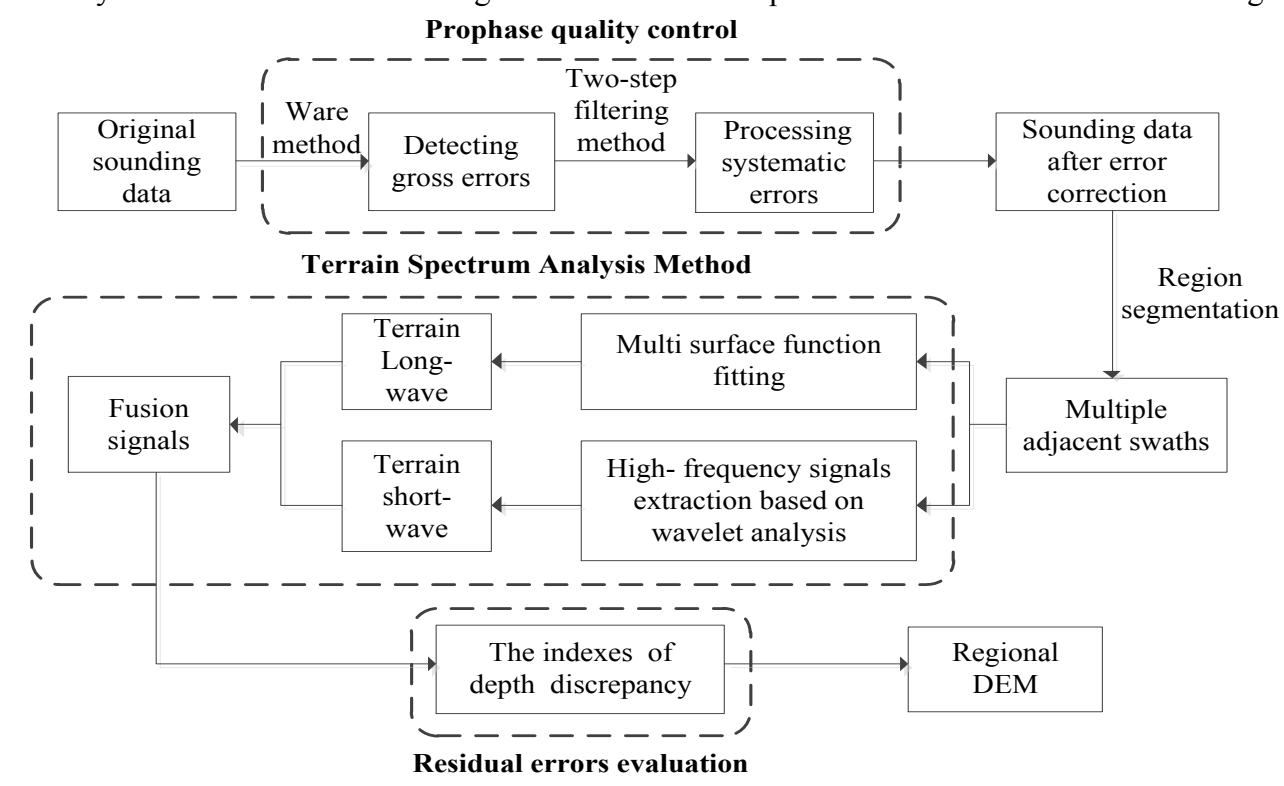

Fig. 1. Research route of weakening the residual errors in swath joins based on terrain spectrum analysis

Note: In fig.1, the prophase quality control involved the gross errors detection by the Ware method and system errors correction by the two-step filtering method; The theories of terrain spectrum analysis included the wavelet analysis to extract the short-wave signals, multi surface function to fit the long wave signals, and the above signals to reconstruct new signals; The residual errors were evaluated by the indexes of the depth discrepancy. 


\subsubsection{Short-wave Signal Exaction}

The residual errors would not affect the subtle changes of terrain features, and thus, the terrain of short-wave signals is credible. Wavelet analysis has the advantage of providing local refinement in the time and frequency domain, and the signal is decomposed into multi scale by telescopic translation operations [25-26]. In this module, Ping is considered a unit. Different wavelet bases, decomposition levels, and threshold values are selected for the quantitative analysis. The selection of optimal wavelet parameters is comprehensively evaluated on the basis of the index results, such as the mean square error (RMSE), signal-to-noise ratio (SNR), and smoothness (S) [27]. A certain Ping original bathymetric signals and their corresponding high-frequency signals extracted by wavelet analysis are shown in Fig. 2.
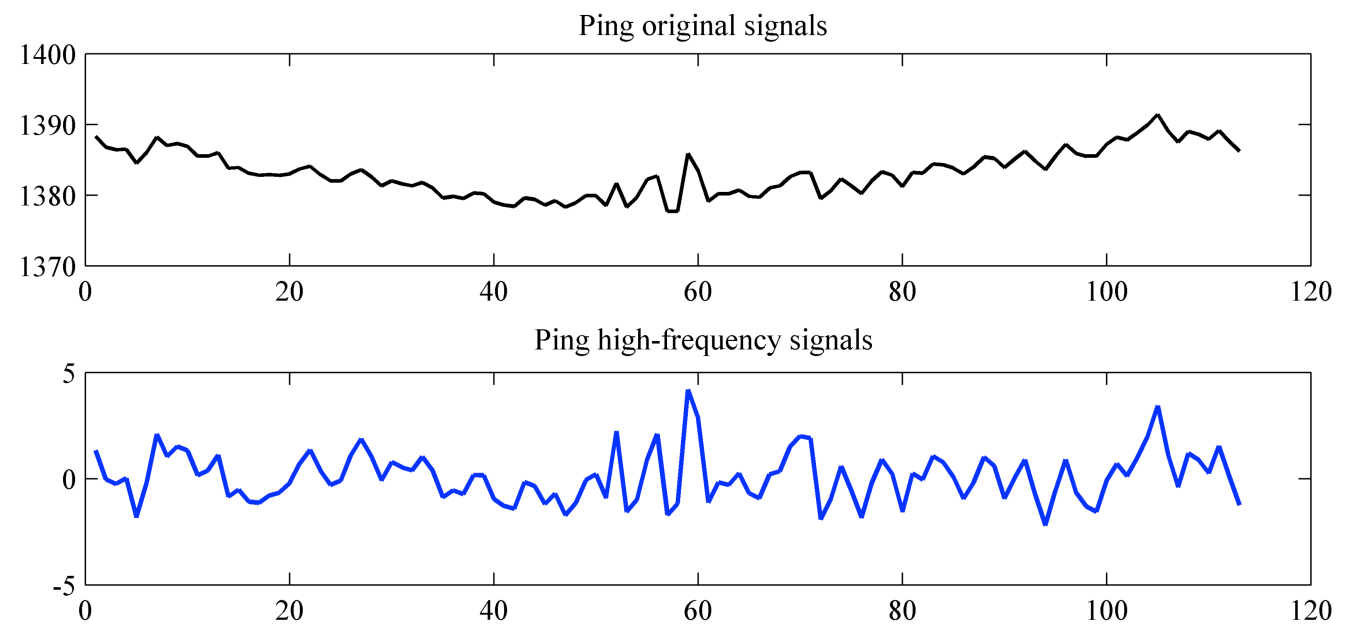

Fig. 2. Extraction of Ping original and high-frequency signals

\subsubsection{Long-wave Signal Exaction}

The sounding data after prophase processing, mixed with the exception of long-periodic signals, cannot reflect the variation of actual seabed topography. However, the quality of sounding data in the central beam area is relatively reliable, and multi-surface function can be applied to fit the main trend of adjacent swaths. As well as, the multi surface function is based on the theory that any regular or irregular continuous surface can be approximated by a number of simple surfaces [28-29]. Each interpolation point establishes a functional relation with all known points, and the contribution values of known points are added [30-31]. Therefore, the superposition value is as the best fitting value of this point. The model is expressed as

$$
S(x, y)=\sum_{i=1}^{m} a_{i} R\left(x, y ; x_{i}, y_{i}\right)
$$

Where

$S(x, y)=$ water depth value of fitting area,

$R\left(x, y ; x_{i}, y_{i}\right)=$ kemel function,

$(x, y)=$ measured coordinates,

$\left(x_{i}, y_{i}\right)=$ coordinates of known points,

$m=$ total number of known points, and

$\alpha_{i}=$ undetermined coefficients.

The kernel function is usually symmetrical.

$$
R\left(x, y ; x_{i}, y_{i}\right)=\left[\left(x-x_{i}\right)^{2}+\left(y-y_{i}\right)^{2}+\delta^{2}\right]^{\beta},
$$

Where $\delta=$ smooth factor, and

$\beta=$ exponential parameter, usually election as $-0.5,0.5$, and 1.5.

The central beam bathymetric data are highly reliable. $n$ data are selected as the measured values, and where here $m$ data are known as nodes. Thus, the form of the matrix is

$\left[\begin{array}{l}S_{1} \\ S_{2} \\ \mathrm{M} \\ S_{n}\end{array}\right]=\left[\begin{array}{cccc}R_{11} & R_{12} & \mathrm{~L} & R_{1 m} \\ R_{21} & R_{22} & \mathrm{~L} & R_{2 m} \\ \mathrm{M} & \mathrm{M} & \mathrm{M} & \mathrm{M} \\ R_{n 1} & R_{n 2} & \mathrm{~L} & R_{n m}\end{array}\right] \cdot\left[\begin{array}{l}\alpha_{1} \\ \alpha_{2} \\ \mathrm{M} \\ \alpha_{m}\end{array}\right] \Rightarrow S=R \alpha$.

When $n$ is not less than $m$,

$V=R \alpha-S \Rightarrow \alpha=\left(R^{T} P R\right)^{-1} R^{T} P S$.

The internal and external fitting accuracy is

$\sigma=\sqrt{[v v] /(n-1)}$

Where

$v=$ difference between the measured value and the fitting calculation value,

$n=$ number of measured values in the central beam, and

$m=$ number of known node.

\subsubsection{Residual Errors Evaluation}

A new signal is synthesized on the basis of the above results of the short-wave and long-wave signals. The effect of residual errors is evaluated by the indexes of depth discrepancy [32-33]. Actually, the probability that a point is 
measured between adjacent swaths is very low, so the method for determining the target point in a pair of Ping is shown in Fig. 3. Assuming the left edge beam point P1 and the water depth $\mathrm{Zp} 1$, then the plane minimum distance point P2 and the water depth $Z_{p_{2}}$ in the corresponding right swath of Ping and the right adjoin Ping (in case Ping pairing is unsuccessful but exists on its adjacent Ping) are ready to be searched. After correction, the depth discrepancy of fused signals is denoted as $\Delta Z_{p}$, and its standard deviation is $S T D$.

$$
\Delta Z p=Z p_{1}-Z p_{2}, S T D=\sqrt{[\Delta Z p \Delta Z p] / n} \text {. }
$$

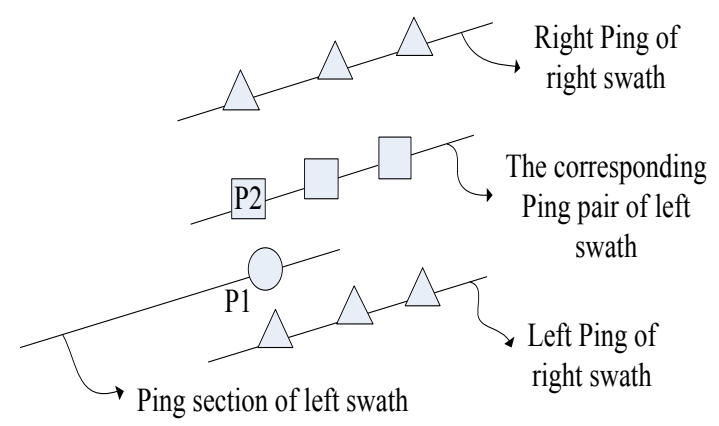

Fig. 3. Method for searching the neighbor target point in a pair of Ping

\section{Result Analysis and Discussion}

The surveyed area is located in the South China Sea. The specific scope is from $16.4^{\circ} \mathrm{N}-17.1^{\circ} \mathrm{N}$ to $109.9^{\circ} \mathrm{E}-110.7^{\circ} \mathrm{E}$. The depth ranges from $1000 \mathrm{~m}$ to $1550 \mathrm{~m}$. The terrain changes slightly with a total of 29 swaths (Fig. 4). The gross point with the property of more than three times the standard deviation is removed by the model of trend surface filter [34] and the system errors in the original sounding data are corrected by two-step filtering method [35]. Finally, the above corrected data are used as the experimental data.

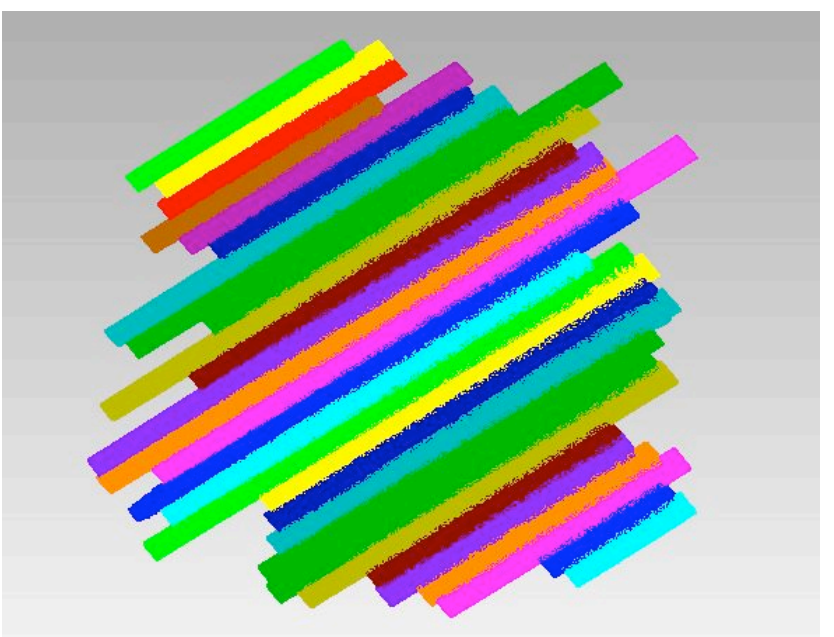

Fig. 4. Distribution map of swath areas

\subsection{Case Study A}

Experiment $\mathrm{A}$ is analyzed using 40 pairs of Ping data between two adjacent swaths. Meanwhile, the optimal parameters of Experiment $\mathrm{A}$ are used as a reference for Experiment B (possess multiple swaths), especially the thinking of optimal node selection in fitting long-wave signals.

The evaluation results of the short-wave signals in Experiment A are displayed in Tab. 1. The smoothness (the smaller, the better) in Table 1 shows that the influence of different wavelet bases and decomposition levels is at millimeter and centimeter scales, so the difference of high frequency signals is fairly small. However, as far as the indexes of RMSE and SNR (the bigger, the better) concern, the best wavelet base coif5 and decomposition of the 3 levels as the best evaluation values at this moment. Thus, the above optimal wavelet parameters are applied to extract the short-wave signals, as same applies to experiment B.

Table 1. Results of different wavelets and decomposition levels with three kinds of evaluation indexes

\begin{tabular}{c|c|c|c|c}
\hline $\begin{array}{c}\text { Wavelet } \\
\text { basis }\end{array}$ & $\begin{array}{c}\text { Decomposed } \\
\text { levels }\end{array}$ & $\begin{array}{c}\text { RMSE } \\
/ \boldsymbol{m}\end{array}$ & SNR & $\begin{array}{c}\text { Smoothnes } \\
\mathbf{s}\end{array}$ \\
\hline Db6 & 3 & 1.0137 & 62.7384 & 0.0720 \\
& 5 & 1.1649 & 61.5351 & 0.0556 \\
\hline Sym4 & 3 & 1.0068 & 62.7977 & 0.0741 \\
& 5 & 1.1568 & 61.5974 & 0.0598 \\
\hline Coif5 & $\mathbf{3}$ & $\mathbf{1 . 0 0 4 3}$ & $\mathbf{6 2 . 8 2 0 2}$ & $\mathbf{0 . 0 7 4 8}$ \\
& 5 & 1.1554 & 61.6074 & 0.0604 \\
\hline
\end{tabular}

When applying the multi surface function to fit the longwave signals, the following three key issues need to be resolved: the number of nodes, the kernel function, and the smooth factor.

(1) Number of nodes. Ping is considered a unit. The data interval for 4-10 points is used as the known node to be researched. Moreover, the fitting accuracy and program running time are used to evaluate the above points. The results are shown in Tab. 2 and Fig. 5. The results indicate that the known node is selected at intervals of 8 points in each Ping central beam area. The program running time is moderate with high fitting precision at this time. Thus, this interval between known nodes can be chosen as the optimal node interval, that is, the number of optimal nodes.

(2) Kernel function. The kernel function between positive hyperbolic and cubic algebraic surface is compared. The specific calculation results are shown in Tab. 3. The fitting accuracy of positive hyperbolic function $(k=0.5)$ is better than that of the three surface kernel functions $(k=1.5)$ in this test area. Therefore, the positive hyperbolic function is the optimal kernel function in this small area.

(3) Smooth factor. Smooth factor affects the matrix rank of normal equation and specifies the relationship between measured point and known node. Moreover, this value must not exceed the scope of the test area. The scope of Experiment $\mathrm{A}$ is $2 * 3 \mathrm{~km}$, thus, the smooth factor values are $100,500,1000$, and 2000 (Table 3). Table 3 shows that the increase in smooth factor also increases the corresponding fitting accuracy. Therefore, the optimal smooth factor is 100 in this area. 
Table 2. Node interval selection and corresponding evaluation values

\begin{tabular}{c|c|c|c|c}
\hline $\begin{array}{c}\text { Node } \\
\text { intervals }\end{array}$ & $\begin{array}{c}\text { Number } \\
\text { of nodes }\end{array}$ & $\begin{array}{c}\text { Running } \\
\text { time }\end{array}$ & $\begin{array}{c}\text { Internal } \\
\text { fitting } \\
\text { precision / } \\
\boldsymbol{m}\end{array}$ & $\begin{array}{c}\text { External } \\
\text { fitting } \\
\text { accuracy / } \boldsymbol{m}\end{array}$ \\
\hline 4 & 1343 & 234.601 & 1.0386 & 1.0590 \\
5 & 1027 & 153.005 & 1.1487 & 1.1951
\end{tabular}

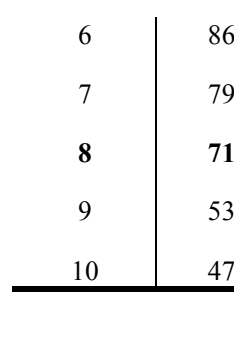

\begin{tabular}{l|r}
869 & 120 \\
790 & 107 \\
711 & 92.6 \\
533 & 68.0 \\
474 & 57.3 \\
\hline
\end{tabular}

\begin{tabular}{l|l|l}
120.916 & 1.2233 & 1.3298 \\
107.890 & 1.2127 & 1.3575 \\
$\mathbf{9 2 . 6 9 5}$ & $\mathbf{0 . 9 8 1 9}$ & $\mathbf{1 . 1 4 4 7}$ \\
68.079 & 1.6548 & 2.0618 \\
57.392 & 1.9361 & 2.4481 \\
\hline
\end{tabular}

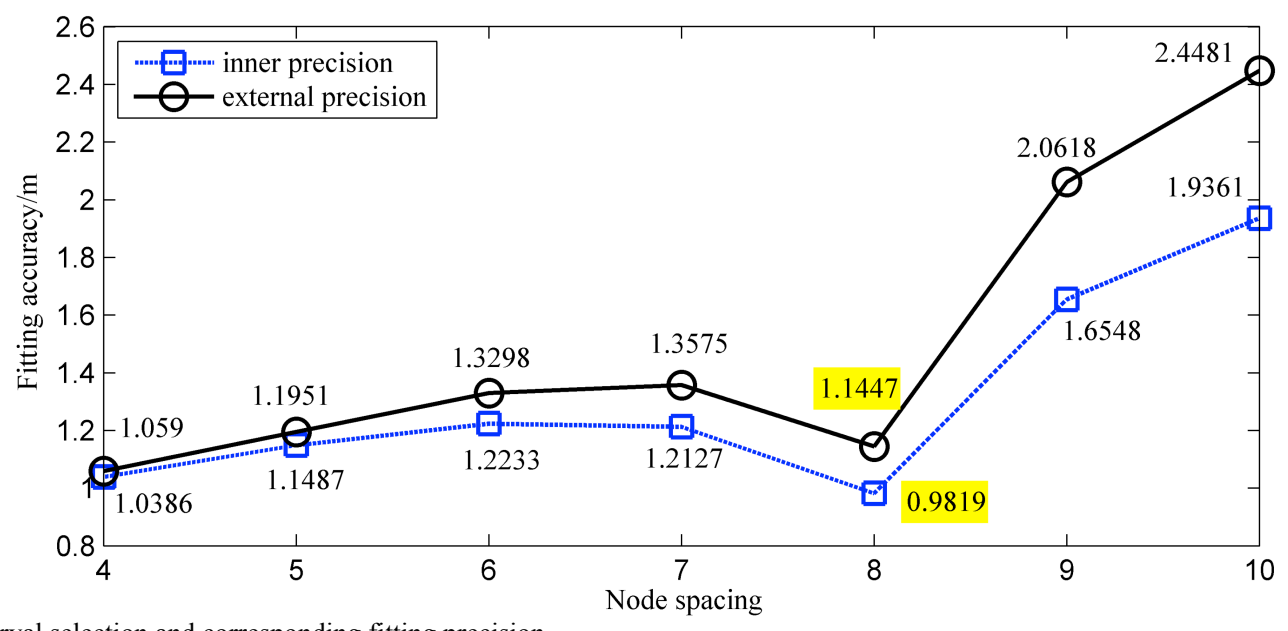

Fig. 5. Data interval selection and corresponding fitting precision

Note: The yellow labels represent the fitting precision of the optimal node interval in the experiment A.

Table 3. Selection of different parameters and corresponding fitting accuracy values with multi-surface function

\begin{tabular}{c|c|c|c|c|c}
\hline Power of kernel $K$ & Smooth factor $\delta$ & $\begin{array}{c}\text { Internode } \\
\text { S }\end{array}$ & Number of nodes & $\begin{array}{c}\text { Internal fitting precision } \\
/ \mathrm{m}\end{array}$ & $\begin{array}{c}\text { External fitting accuracy } \\
/ \mathrm{m}\end{array}$ \\
\hline \multirow{5}{*}{$\mathbf{0 . 5}$} & 100 & 5 & 1027 & 1.1487 & 1.1951 \\
& $\mathbf{1 0 0}$ & $\mathbf{8}$ & $\mathbf{7 1 1}$ & $\mathbf{0 . 9 8 1 9}$ & $\mathbf{1 . 1 4 4 7}$ \\
& 100 & 9 & 533 & 1.6548 & 2.0618 \\
& 500 & 8 & 711 & 1.1765 & 1.2754 \\
& 1000 & 8 & 711 & 1.2576 & 1.3239 \\
\hline \multirow{5}{*}{1.5} & 100 & 6 & 869 & 1.2484 & 1.2917 \\
& 100 & 8 & 711 & 1.1783 & 1.2834 \\
& 100 & 9 & 533 & 1.3260 & 1.3321 \\
& 1000 & 5 & 1027 & 1.3839 & 1.3885 \\
& 1000 & 8 & 711 & 1.3216 & 1.3881 \\
\end{tabular}

Note: Compared to other parameters, the bold marks showed that the fitting accuracy is optimal in the experiment A.

After the analysis of above optimal parameters with multi surface function, the terrain long-wave fitting signals are acquired [Fig. 6(b)]. The results show that the terrain changes, which are reflected by the long-wave signals of the edge beam, are consistent with the central beam terrain trend. These signals can effectively eliminate the uplift phenomenon between swaths and can verify the feasibility of parameter selections with multi-surface function. 


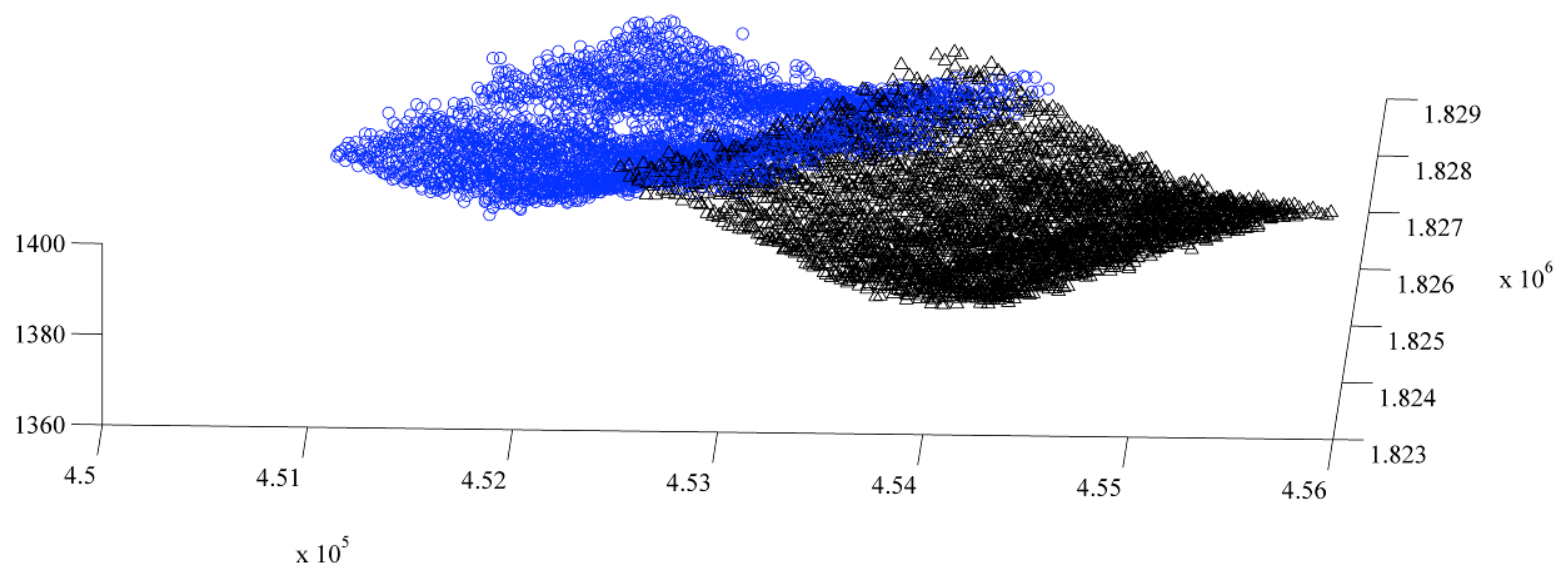

(a) Original sounding signals of local areas

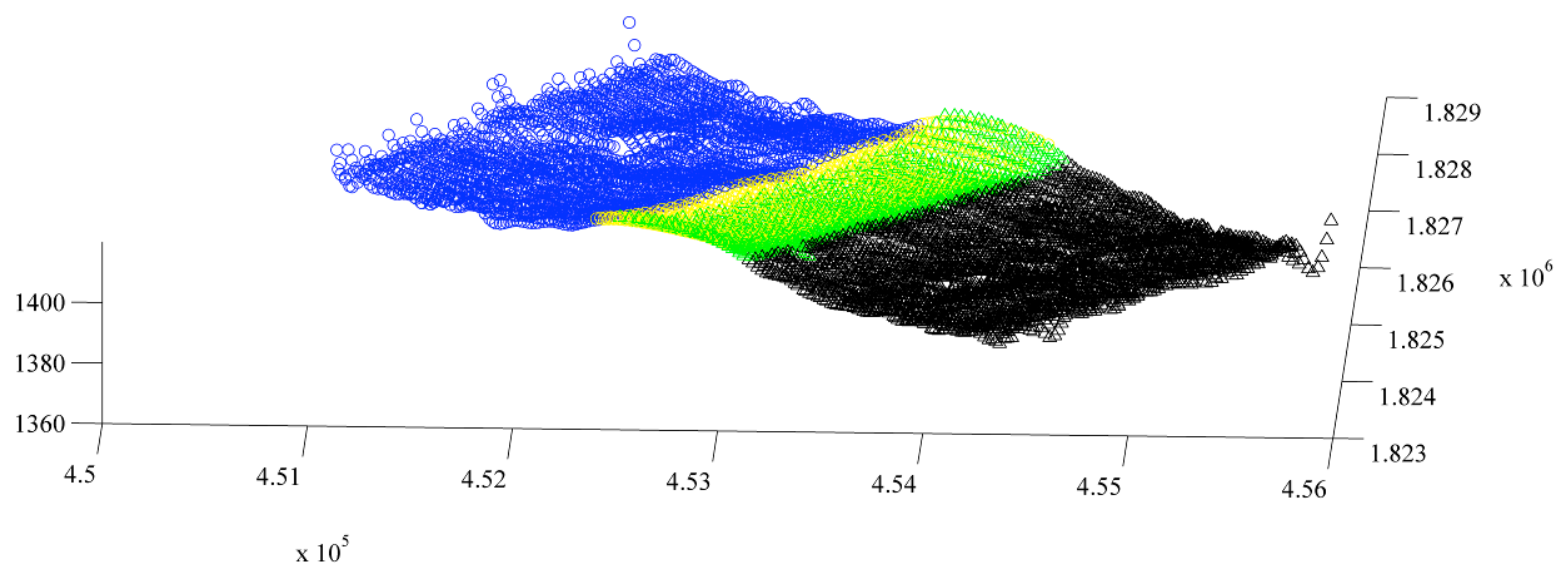

Fig. 6. Terrain long-wave signal extraction effect

(b) Fitting signals based on multi-surface function

\subsection{Case Study B}

In Experiment A, only some pairs of Ping data are selected. However, the entire surveyed area is composed of more than 40 pairs of Ping data. Such large data can result in timeconsuming and inefficient calculation with similar the treatment of experimental A. Moreover, if the adjacent swaths are treated as a whole, the normal equation will be large, and measured point correlation can be significant. This condition will cause loss of rank and low calculation accuracy, even for the normal equation cannot be resolved. Therefore, the surveyed area is divided into 8 kinds of large areas to extract the terrain long-wave signals. The width of swaths is usually larger than $10 \mathrm{~km}$, thus, the optimal parameters of multi-surface function are selected by the above test ideas. The optimal smooth factor for a large area is 10000 . Kernel function is the positive hyperbolic function through repeated experiments. Meanwhile, one in five Ping is selected, five points are chosen as the known node in the central beam area of this Ping. Good results in fitting accuracy, running time, and size of normal equation are thus expected.

The corresponding long-wave signals are extracted in 8 pieces of areas. Consequently, the fitting error, node number, and running time in each area are obtained. The statistics in Tab. 4 show that overlapping swaths exist in each area, which can ensure the consistent trend of communal swath terrain. Using the optimal parameter selection, the fitting error is constantly controlled in around $2.0 \mathrm{~m}$, and the relative precision of bathymetric is $14-18 \mathrm{~mm}$. These values meet the accuracy requirements of deep water area.

Table 4. Statistical parameters of the entire area

\begin{tabular}{c|c|c|c|c}
\hline $\begin{array}{c}\text { Surveyed } \\
\text { area }\end{array}$ & $\begin{array}{c}\text { Overlapping } \\
\text { swath } \\
\text { number }\end{array}$ & $\begin{array}{c}\text { Node } \\
\text { number }\end{array}$ & $\begin{array}{c}\text { Fitting } \\
\text { errors/m }\end{array}$ & $\begin{array}{c}\text { Running } \\
\text { time }\end{array}$ \\
\hline Area 1 & $1-5$ & 702 & $\mathbf{1 . 7 6 6 8}$ & 145.383 \\
Area 2 & $5-9$ & 1142 & $\mathbf{2 . 0 2 4 6}$ & 409.135 \\
Area 3 & $9-12$ & 1141 & $\mathbf{2 . 0 0 7 6}$ & 403.202 \\
Area 4 & $12-15$ & 1312 & $\mathbf{2 . 0 6 2 0}$ & 631.302 \\
Area 5 & $15-18$ & 1406 & $\mathbf{2 . 0 3 9 4}$ & 727.538 \\
Area 6 & $18-21$ & 1227 & $\mathbf{2 . 0 9 5 0}$ & 559.235 \\
Area 7 & $21-25$ & 1165 & $\mathbf{2 . 0 6 8 4}$ & 455.441 \\
Area 8 & $25-29$ & 903 & $\mathbf{2 . 0 7 8 0}$ & 261.738 \\
\hline
\end{tabular}


Note: In tab.4, the bold marks are the fitting accuracy of each block area in the process of extracting the long-wave signals.

The terrain short-wave and long-wave signals are integrated into a new signal. The consistency of the sounding data of overlapping areas is evaluated to analyze the effect of residual errors. The effect of residual error in the local area of Experiment $\mathrm{A}$ is compared with the results of Experiment B (Tab. 5). The contour map and original seabed DEM present an uneven terrain in the entire areas [Fig. 7(a) and Fig. 8(a)]. However, the corrected contour map and DEM remain smooth and continuous [Fig. 7(b) and Fig. 8(b)]. The uplift phenomenon can be effectively eliminated, and the subtle changes of terrain seabed can be reasonably reflected in the surveyed area.

Tab. 5. Statistic evaluation parameters of residual errors

\begin{tabular}{|c|c|c|c|c|c|}
\hline Experiment & Correction state & $\begin{array}{c}\text { Maximum } \\
\text { deviation } / m\end{array}$ & $\begin{array}{c}\text { Minimum } \\
\text { deviation } / m\end{array}$ & Mean value/m & $\begin{array}{c}\text { Standard } \\
\text { deviation } / m\end{array}$ \\
\hline \multirow{2}{*}{$\begin{array}{l}\text { A (40 pairs of Ping data between two } \\
\text { adjacent swaths) }\end{array}$} & Before & 8.0000 & -11.2000 & -0.8674 & 3.0520 \\
\hline & After & 5.6064 & -4.7915 & -0.0263 & 1.6434 \\
\hline \multirow[t]{2}{*}{ B (all adjacent swaths) } & Before & 18.8049 & -19.7677 & -1.9730 & 4.4015 \\
\hline & After & 6.3680 & -7.2373 & -0.0705 & 1.9400 \\
\hline \multirow{2}{*}{$\begin{array}{l}\text { The DFT method } \\
\text { (all adjacent swaths) }\end{array}$} & Before & -21.711 & 36.560 & 2.859 & 4.730 \\
\hline & After & -9.992 & 14.937 & 0.704 & 2.645 \\
\hline
\end{tabular}

Note: In tab. 5, the data source in this paper is the same as in the DFT method. The evaluation values of the DFT (discrete Fourier transform) method derived from the tab. 2 in the literature [20]. However, the DFT method is not to remove the gross errors and correct the system errors before correction, so the evaluation values are different between the experiment B and its method.

Tab. 5 shows that the weakening effect of residual errors in experiment $\mathrm{A}$ is better. However, the large area of multiple swaths can quickly process in experiment B. Appropriately decreasing the accuracy can still achieve the residual error reduction. Meanwhile, based on the same data source, the method of this paper is obviously better than the DFT method. The mean values show that a systematic error exists in the depth data before correction, but the mean of synthetic signal is close to 0 after correction. The corrected depth is in accordance with the Gauss distribution. This similarity indicates that the method can effectively reduce

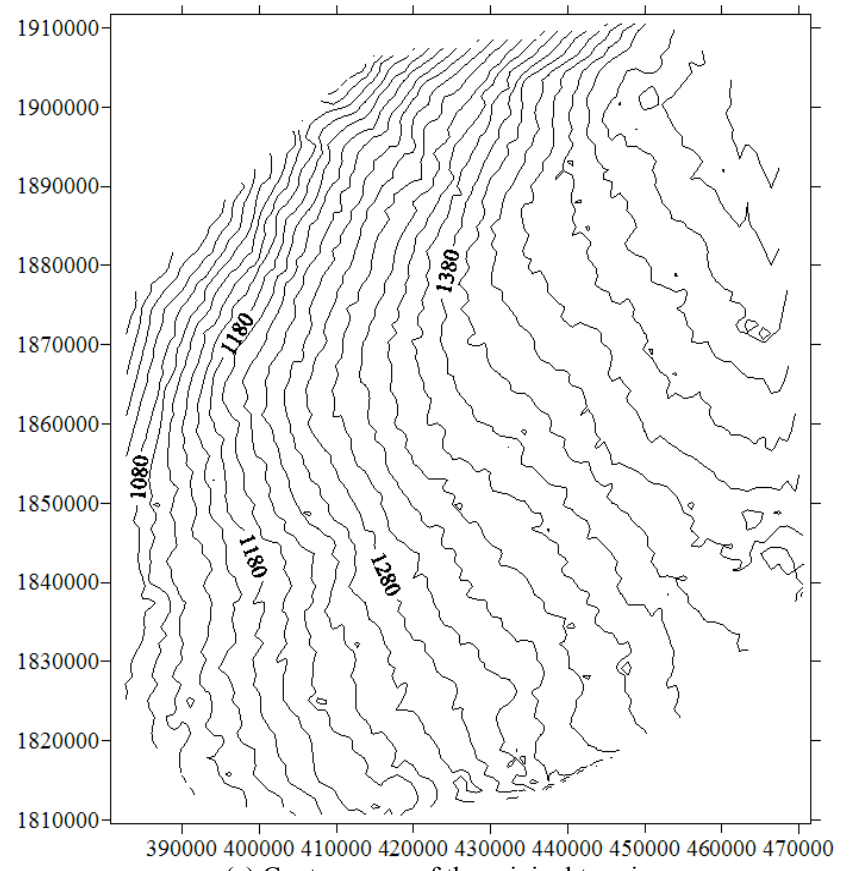
(a) Contour map of the original terrain

Fig. 7. Comparison effects of contour map the systematic residual error. Moreover, for the standard deviation and extreme values, the convergent of depth discrepancy is improved at the edge of adjacent swaths after correction. However, a few points still exist around 6-7 m bias, because the experiment is based on the hypothesis that the sounding data quality is reliable in central beam area. Nevertheless, some redundant points remain in the bathymetric data, these excess points are difficult to be eliminated and affect the quality of the experiment. Therefore, the abnormal values of water depth must be removed before the experiment.

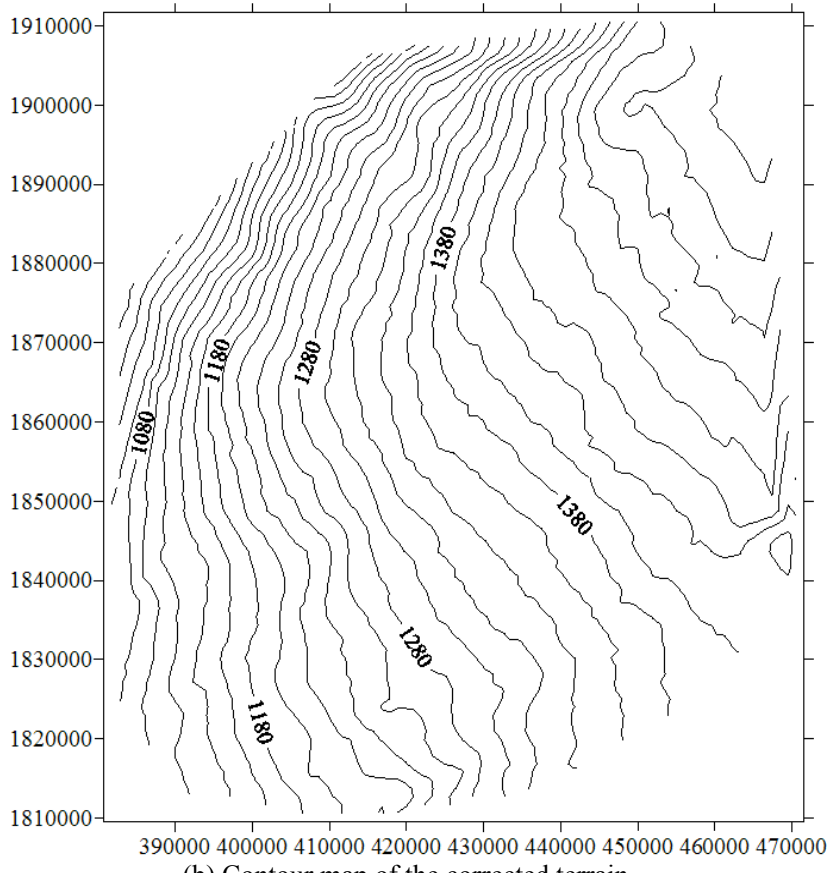

(b) Contour map of the corrected terrain 


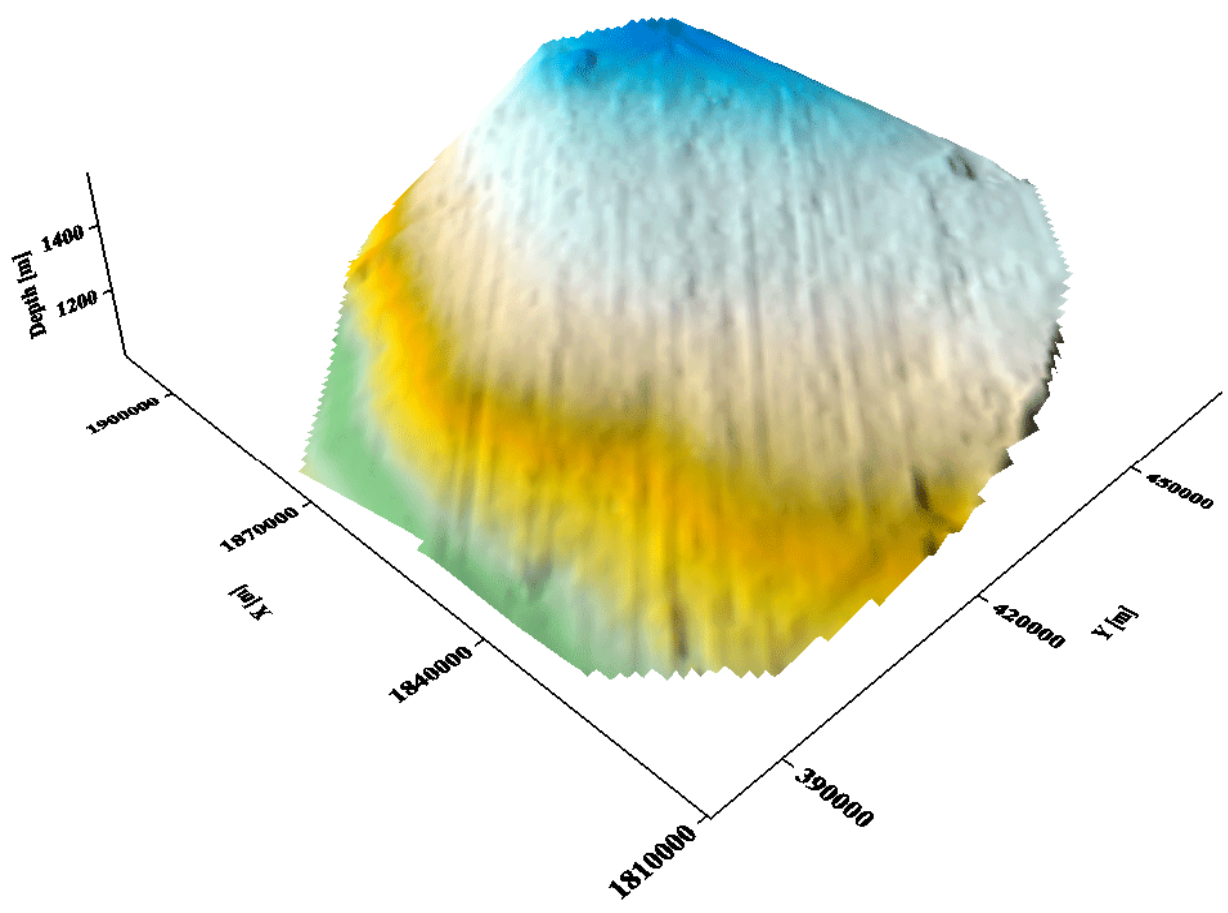

1550

$-1500$

$-1450$

$-1400$

$-1350$

$-1300$

$-1250$

$-1200$

$-1150$

$-1100$

$-1050$

(a) Original terrain DEM

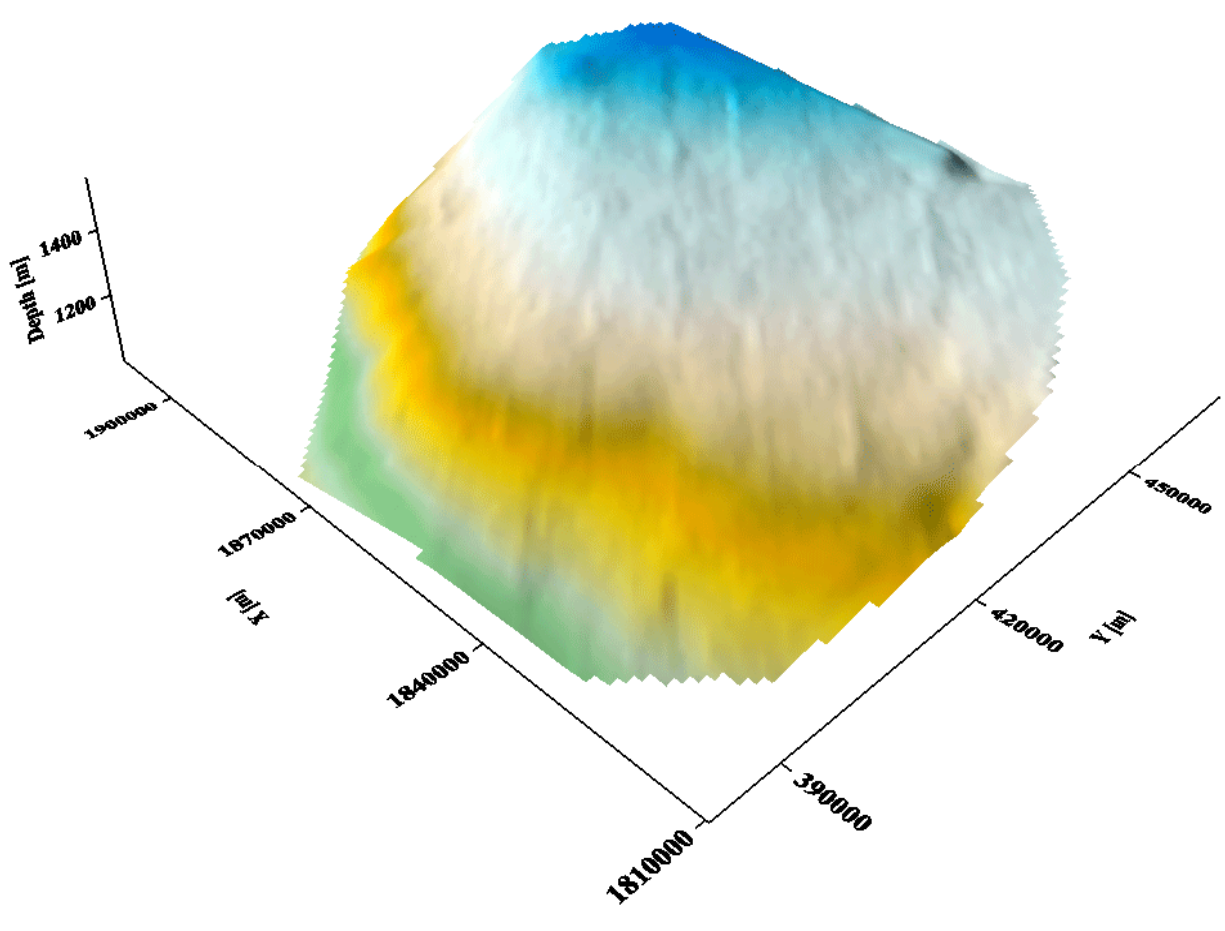

1550

$-1500$

$-1450$

$-1400$

$-1350$

$-1300$

$-1250$

$-1200$

$-1150$

$-1100$

$-1050$

(b) Corrected terrain DEM

Fig. 8. Comparison effects of investigative areas DEM

Note: Seabed DEM (digital elevation model) generated by Golden Surfer software using all of the swaths; The gridding is achived by the interpolation method of triangulation with linear; In order to meet the width of the text, and improve the aesthetics, This figure changes the font and size of the axis; Morever, the corrected DEM effect can be compared with the fig. 14(b) of the literature [20] (the source of original data is the same, just the coordinates are the value of Latitude and Longitude in fig.14(b)). the experimental results in this paper is obviously better than the result of the DFT ( discrete Fourier transform).

\section{Conclusions}

For sounding data, this paper firstly removed the gross errors and corrected the system errors. Two study examples are then established to compare the efficiency and practicability with the proposed method. Moreover, its reliability was evaluated by the indexes of depth discrepancy. The main conclusions of this study are shown as follows:

1) In Experiment A, the estimates of residual error are better, but the operation efficiency is low for massive data. In Experiment B, the method of appropriately reducing the fitting accuracy is adopted to quickly process the sounding 
data. As a result, Experiment B also achieves good residual error weakening.

2) Unlike traditional methods, the proposed method needs no the continuous matching of Ping pair data and also reduces the possibility of a mismatch. The multi-surface function module can obtain the fitting signals of nonoverlapping areas. Moreover, the evaluation indexes of optimal parameters can effectively assess the short-wave and long-wave signals.

3) A few gross errors remain in the central beam area. These errors distract the fitting accuracy of multi-surface function and yield the high-frequency signals extracted by wavelet analysis with noise signals. This phenomenon results in a few large extreme deviations (minimum or maximum) of depth discrepancy after correction. Therefore, it is also needed to deal with the sounding data of the central beam area through a certain method for detecting gross errors before correction, which is the research content of the further work.

\section{Acknowledgements}

This study was supported by the Doctor Start-up Fund of the East China Institute of Technology (DHBK2012204), the National Natural Science Foundation of China (41206078, 41404026), and the Director Fund of the Second Institute of Oceanography (JG-1508).

\section{References}

1. LIU Jingnan, ZHAO Jianhu., "Present Status and Development Trend of Multibeam Sounding System", Hydrographic Surveying and Charting, 22(5), 2002, pp. 3-6.

2. Geng, Xueyi, Zielinski, Adam., "Precise Multibeam Acoustic Bathymetry", Marine Geodesy, 22(3), 1999, pp.157-167.

3. Verner B. Ernstsen, Riko Noormets, Dierk Hebbeln, et al., "precision of High-resolution Multibeam Echo Sounding Coupled with High-accuracy Positioning in a Shallow Water Coastal Environment", Geo-Marine Letter, 26(3), 2006, pp.141-149.

4. Le Bas, T.P, Somers, M.L, Campbell, J.M, et al., "Swath Bathymetry with GLORIA", IEEE Journal of Oceanic Engineering, 21(4), 1996, pp.545-553.

5. Xavier Lurton., "Theoretical Modelling of Acoustical Measurement Accuracy for Swath Bathymetric Sonars", The International Hydrographic Review, 4(2), 2003, pp.17-30.

6. Godin A, Crutchlow MR., "Swath Sounding Initiatives in Canada", International Hydrographic Review, 75(1), 1998, pp.65-80.

7. Herlihy DR, Stepka TN, Rulon TD., "Filtering Erroneous Soundings from Multibeam Survey Data", International Hydrographic Review, 69(2), 1992, pp.67-72.

8. Larry A. Mayer., "Frontiers in Seafloor Mapping and Visualization", Marine Geophysical Researches, 27(1), 2006, pp.717.

9. P. R. Ogushwitz, P. S. Dysart., "Computer Simulation of Multibeam Echo Sounding over Rough Seafloor", Marine Geodesy, 15(2-3), 1992, pp.97-113.

10. Bishwajit Chakraborty, Vijay N. Kodagali., "Seabottom Roughness Study Using a Hydrosweep--Multibeam System", The Journal of the Acoustical Society of America, 105(2), 1999, pp.1266.

11. HARE R., "Depth and Position Error Budgets for Multibeam Echosounding", International Hydrographic Review, 72(2), 1995, pp.37-69.

12. B. R. Calder, L. A. Mayer., "Automatic Processing of High-rate, High-density Multibeam Echosounder Data", G-Cubed: Geochemistry, Geophysics, Geosystem, 4(6), 2003, pp.1048-1069.

13. Bjorke J.T., "Computation of Calibration Parameters for Multibeam Echo Sounders Using the Least Squares Method", IEEE Journal of Oceanic Engineering, 30(4), 2005, pp.818-831.

14. ZOU Yonggang, CAI Shu, ZHU Xiaochen, et al., "A New Method for Multibeam Swath Join", Twenty-one Symposium on Marine Surveying and Mapping, 2009.

15. HUANG Motao, ZHAI Guojun, OUYANG Yongzhong, et al., "Data Fusion Technique for Single Beam and Multibeam Echosoundings", Acta Geodaetica et Cartographica Sinica, 30(4), 2001, pp.299-303.

16. LI Mingsan, SUN Lan, SUN Qiang, et al., "Adjustment Model Based on Ping Sructure for Swath Combination Net", Geomatics and Information Science of Wuhan University, 36(6), 2011, pp.652655 .

17. WANG Hongyan, LI Mingsan, SUN Qiang, et al., "A Quick Algorithm for Calculating Parameters of Swath Combination Net", Hydrographic Surveying and Charting, 34(5), 2014, pp.31-33.

18. WU Ziyin, JIN Xianglong, ZHENG Yulong, et al., "Integrated Error Correction of Multi-beam Marginal Sounding Beam", Journal of Ocean (Chinese Version), 27(4), 2005, pp.88-94.
19. ZHAO Jianhu, ZHANG Hongmei, YAN Jun, et al., "Weakening Influence of Residual Error for MBS Sounding", Geomatics and Information Science of Wuhan University, 38(10), 2013, pp.11841187.

20. Jianhu Zhao, Jun Yan, Hongmei Zhang, et al., "A New Method for Weakening the Combined Effect of Residual Errors on Multibeam Bathymetric Data”, Marine Geophysical Researches, 35(4), 2014, pp.379-394.

21. Neil C. Mitchell., "Processing and Analysis of Simrad Multibeam Sonar Data", Marine Geophysical Researches, 18(6), 1996, pp.729739 .

22. David W. Caress, Dale N. Chayes., "Improved Processing of Hydrosweep DS Multibeam Data on the R/V Maurice Ewing", Marine Geophysical Researches, 18(6), 1996, pp.631-650.

23. Ware C, Knight W, Wells D., "Memory Intensive Satistical Algorithms for Multibeam Bathymetric Data", Computer and Geosciences, 17(7), 1991, pp.985-993.

24. ZHAO Jianhu, LIU Jingnan., "Multi-beam Sounding and Image Data Processing”, Wuhan: Wuhan University Press, China, pp.210214.

25. LIU Yunjie, LIU Yihui., "Wavelet High-frequency Coefficients for Feature Extraction of Gene Microarray Data", China Journal of Bioinformatics, 9(4), 2011, pp.339-343.

26. WU Wenfeng, LIU Yihui., "Feature Selection for Highdimensional Protein Mass Spectrometry Data Based on the High Frequency Coefficients of Wavelet Analysis", China Journal of Bioinformatics, 13(3), 2015, pp.198-204.

27. TAO Ke, ZHU Jianjun., "A Comparative Study on Validity Assessment of Wavelet De-noising”, Journal of Geodesy and Geodynamics, 32(2), 2012, pp.128-133.

28. Rolland L. Handy., "Multiquadric Equations of Topography and Other Irregular Surfaces", Journal of Geophysical Research, 76(8), 1971, pp.1905-1915.

29. M. Schechter., "Polyhedral Functions and Multiparametric Linear Programming", Journal of Optimization Theory and Applications, 53(2), 1987, pp.269-280.

30. ZHANG Jie, BAO Jingyang, XU Jun, et al., "The Application of the Hardy Function in the Creation of Regional Tidal Model", Geomatics \& Spatial Information Technology, 35(5), 2012, pp.4-7.

31. ZHOU Mingming, WANG Shandong, WANG Lu., "GPS Height Fitting Based on Conicoid Function and Multi-faceted Function", Computer \&Digital Engineering, 42(2), 2014, pp.315-318.

32. Brian R. Calder., "On Risk-Based Expression of Hydrographic Uncertainty", Marine Geodesy, 38(2), 2015, pp.99-127.

33. Brian R. Calder., "Multi-Algorithm Swath Consistency Detection for Multibeam Echosounder Data", The International Hydrographic Review, 8(1), 2007, pp.9-25.

34. DONG Jiang, Ren Lisheng., "Filter of MBS Sounding Data Based on Trend Surface", Hydrographic Surveying and Charting, 27(6), 2007, pp.25-28.

35. ZHAO Jianhu, LIU Jingnan, YANG Fanlin., "Weaken Systematic Error in Depth Data of MES", Geomatics and Information Science of Wuhan University, 29(5), 2004, pp.394-397. 